Document downloaded from:

http://hdl.handle.net/10251/94536

This paper must be cited as:

Crespo-Poveda, A.; Hernández-Mínguez, A.; Biermann, K.; Tahraoui, A.; Gargallo-Jaquotot, B.; P. Muñoz; Santos, P.... (2016). Tunable Interferometers Driven by Coherent Surface Acoustic Phonons. MRS Advances. 1651-1656. doi:10.1557/adv.2016.234

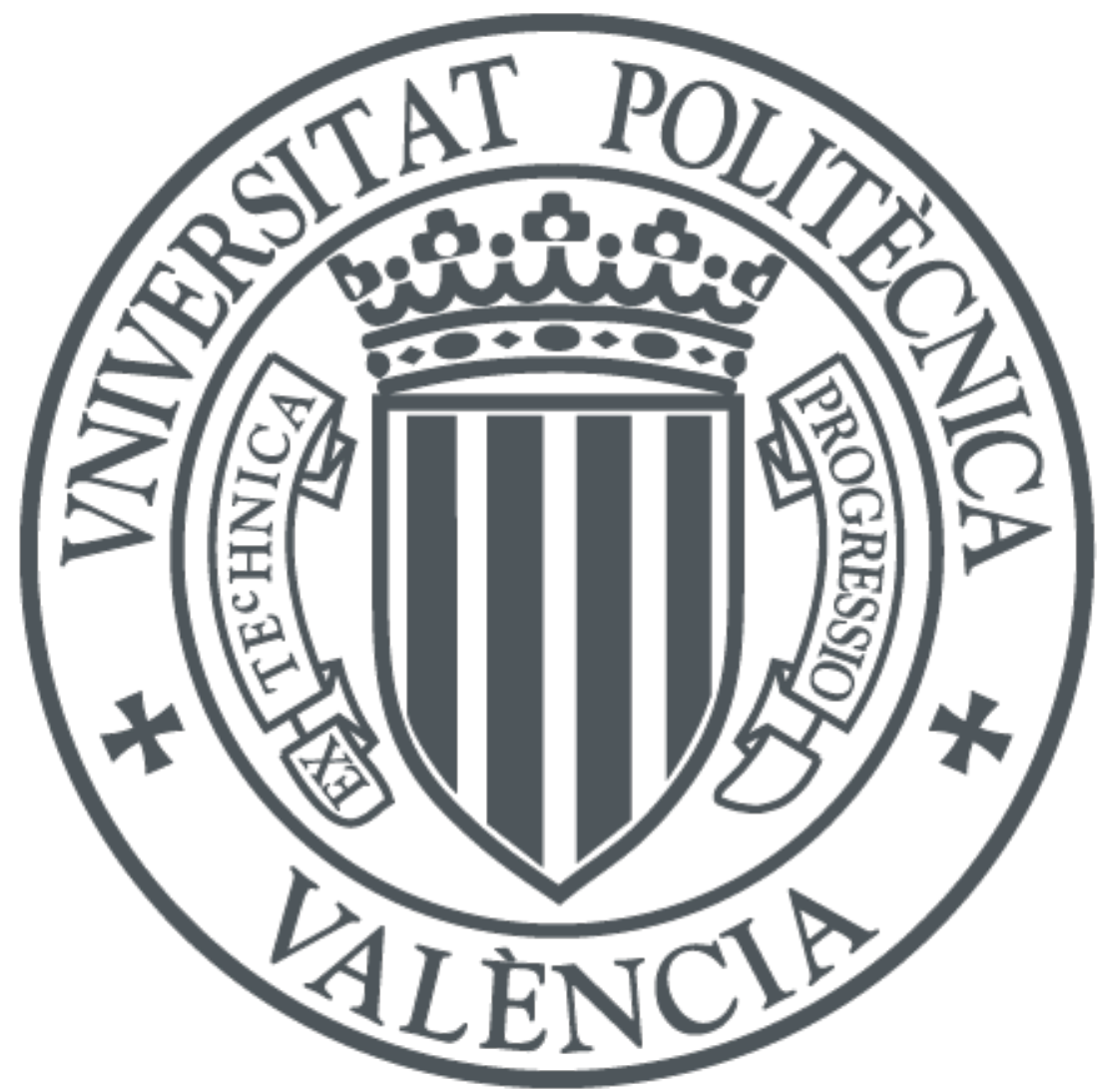

The final publication is available at

https://doi.org/10.1557/adv.2016.234

Copyright Cambridge University Press (Materials Research Society)

Additional Information 


\section{MRS Advances}

\section{Tunable Interferometers Driven by Coherent Surface Acoustic Phonons}

\begin{tabular}{|r|l|}
\hline Journal: & 2015 MRS Fall Meeting \\
\hline Manuscript ID & Draft \\
\hline Manuscript Type: & Symposium II \\
\hline Date Submitted by the Author: & n/a \\
\hline Complete List of Authors: & $\begin{array}{l}\text { Crespo-Poveda, Antonio; Materials Science Institute University of Valencia } \\
\text { Hernández-Mínguez, Alberto; Paul-Drude-Institut für Festkörperelektronik, } \\
\text { Biermann, Klaus; Paul-Drude-Institut für Festkörperelektronik } \\
\text { Tahraoui, Abbes; Paul-Drude-Institut für Festkörperelektronik } \\
\text { Gargallo, Bernardo; iTEAM Research Institute Polytechnical University Of } \\
\text { Valencia } \\
\text { Muñoz, Pascual; iTEAM Research Institute Polytechnical University Of } \\
\text { Valencia } \\
\text { Santos, Paulo; Paul-Drude-Institut für Festkörperelektronik, } \\
\text { Cantarero, Andrés; Institut de Ciència dels Materials, Universitat de } \\
\text { València, } \\
\text { de Lima, Jr., Mauricio; Institut de Ciència dels Materials, Universitat de } \\
\text { València, }\end{array}$ \\
\hline Keywords: & \begin{tabular}{l} 
devices, optoelectronic, acoustic \\
\hline \hline
\end{tabular} \\
\hline \hline
\end{tabular}




\title{
Tunable Interferometers Driven by Coherent Surface Acoustic Phonons
}

\author{
Antonio Crespo-Poveda, ${ }^{1}$ Alberto Hernández-Mínguez, ${ }^{2}$ Klaus Biermann, ${ }^{2}$ Abbes Tahraoui, ${ }^{2}$ \\ Bernardo Gargallo, ${ }^{3}$ Pascual Muñoz, ${ }^{3}$ Paulo V. Santos, ${ }^{2}$ Andrés Cantarero, ${ }^{1}$ and Maurício M. de \\ Lima, Jr. ${ }^{1}$ \\ ${ }^{1}$ Materials Science Institute, University of Valencia, P.O. Box 2208, ES46071 Valencia, Spain \\ ${ }^{2}$ Paul Drude Institute for Solid State Electronics, Hausvogteiplatz 5-7, DE10117 Berlin, \\ Germany \\ 3 iTEAM Research Institute, Polytechnic University of Valencia, Camino de Vera s/n, ES46022 \\ Valencia, Spain
}

\begin{abstract}
We demonstrate a compact tunable photonic modulator driven by surface acoustic waves (SAWs) in the low GHz frequency range. The device follows a well-known Mach-Zehnder interferometer (MZI) structure with three output channels, built upon multi-mode interference (MMI) couplers. The light continuously switches paths between the central and the side channels, avoiding losses and granting a $180^{\circ}$-dephasing synchronization between them. The modulator was monolithically fabricated on $(\mathrm{Al}, \mathrm{Ga}) \mathrm{As}$, and can be used as a building block for more complex photonic functionalities. It can also be implemented in other material platforms such as Silicon or (In,Ga)P. Light modulated at multiples of the fundamental acoustic frequency can be accomplished by adjusting the applied acoustic power. An excellent agreement between theory and experiment is achieved.
\end{abstract}

\section{INTRODUCTION}

A great effort has been made during the past decades to increase the integration level in photonic circuits. An incipient exponential trend in the number of integrated elements per chip resembling the well known Moore's law can be appreciated. Although integration levels of up to a few thousands of components per chip have been accomplished [1], the absence of a standarized integration technology has prevented so far photonic integrated circuits from reaching the same development followed by its electronic counterpart. It is then of superlative importance to search for devices that are at the same time compatible with nowadays integration technology, fast, compact and inexpensive. The external control of light propagation inside the devices allows also for a reduction in the dimensions, favouring the integration and giving rise to novel functionalities. A promising approach consists of using coherent surface acoustic phonons in the form of a SAW to modulate single or multiple ridge or slot waveguides (WGs) through the acousto-optical effect [2-5], with the possibility of addressing several devices with the same SAW beam. This method presents an excellent compromise between speed and size, and can be implemented in almost any material platform such as Silicon, (In, Ga)P or $\mathrm{LiNbO}_{3}$. In this contribution, we present a three output photonic modulator driven by SAWs in the low $\mathrm{GHz}$ 
frequency range, in which the light is continuously split, with a $180^{\circ}$-dephased synchronization, between the central and side WGs.

\section{THEORY}

\section{$\underline{\text { Device concept }}$}

The device consists of a MZI structure with three output WGs, built upon MMI couplers with the same coupling length (a sketch of the fabricated device can be seen in Figure 1). The first coupler is a balanced (50:50) splitting ratio MMI coupler with a single access WG that splits the incoming light into two identical optical beams which are then modulated by a SAW beam propagating perpendicularly to them $\left(\mathrm{MWG}_{1}\right.$ and $\left.\mathrm{MWG}_{2}\right)$. The SAW beam is generated by an interdigital transducer (IDT). A second MMI coupler combines the input light into three output WGs $\left(\mathrm{OC}_{1}, \mathrm{OC}_{2}\right.$ and $\mathrm{OC}_{3}$, respectively) with different power distribution depending on the phase and intensity of the light coming from the active region of the device. The design of the MMI couplers ensures that all the light is directed to $\mathrm{OC}_{2}$ in the absence of acoustic excitation. By introducing a phase difference $\Delta \Phi=\delta \Phi_{\mathrm{MWG} 1}-\delta \Phi_{\mathrm{MWG} 2}$ between the MWGs, the output power can be fully directed to $\mathrm{OWG}_{2}$ as in the absence of SAWs, (for $\Delta \Phi=0$, or equivalently $\Delta \Phi=$ $\pm k \pi, k=1,2,3 \ldots$ ) or equally distributed between $\mathrm{OC}_{1}$ and $\mathrm{OC}_{3}$ (for $\Delta \Phi= \pm k \pi / 2, k=1,3,5 \ldots$ ). The phase difference $\Delta \Phi$ arises from the SAW modulation via the acousto-optic and the electrooptic effect, associated with the strain and piezoelectric fields, respectively. The acousto-optic effect dominates in the situation we discuss here. The spatial separation between the WGs in the active region of the device is a crucial aspect to achieve such a phase difference between the optical beams leaving the MWGs, and must be set to $(2 \mathrm{~m}+1) \lambda_{\mathrm{SAW}} / 2[2-4]$, where $\lambda_{\mathrm{SAW}}$ is the

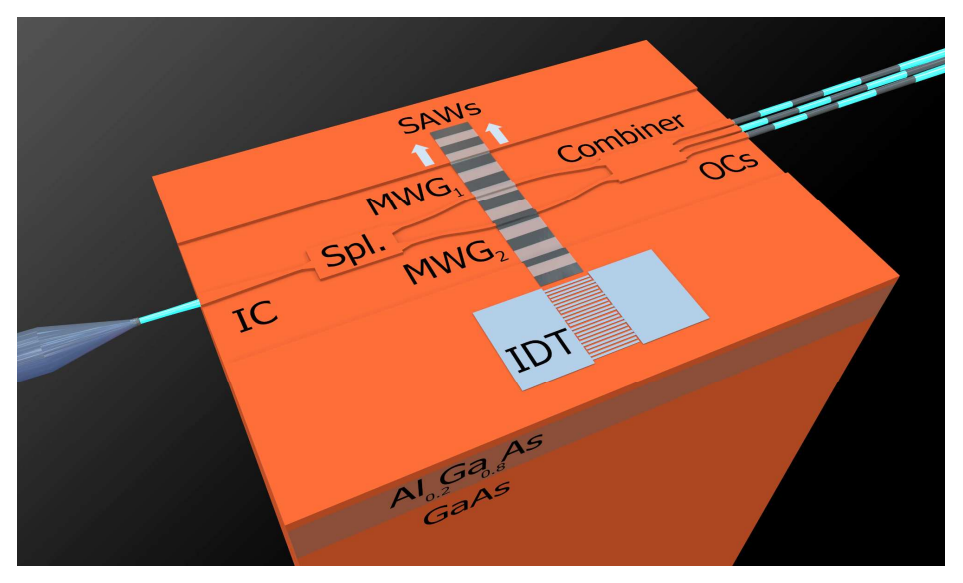

Figure 1. Illustration (not to scale) of the SAW-driven Mach-Zehnder interferometer with three output channels, fabricated on (A1,Ga)As. The light is coupled into an input channel (IC) by means of an optical fiber probe. The device consists of a splitter (Spl.) and combiner multi-mode interference (MMI) couplers of equal length linked by waveguides (MWGs) that are modulated by a SAW, generated by an interdigital transducer (IDT), and propagating perpendicularly to them. The light beams leaving the device through output channels $\mathrm{OC}_{1,3}$ and $\mathrm{OC}_{2}$ present a $180^{\circ}-$ dephasing synchronization. 
SAW wavelength and $\mathrm{m}$ is an integer. This ensures that each of the MWGs suffers a change in the effective refractive index of equal magnitude but opposite phase, maximizing therefore the acousto-optical modulation. The MWGs are placed at the appropriated positions by means of large radius S-bend WGs, minimising the width of the MMI couplers (and consequently, the dimensions of the device) without introducing undesirable phase changes. The magnitude of the phase change introduced by the SAW in each of the MWGs in the active region can be expressed as $[2,4,5]$ :

$$
|\delta \Phi|=\frac{2 \pi \ell}{\lambda}\left|\delta n_{e f f}\right|=a_{p} \sqrt{P_{I D T}}
$$

where $\ell$ is the interaction length between the light and the acoustic field in the active region, $\lambda$ is the light wavelength, $a_{p}$ is a proportionality constant that depends on the elasto-optical properties of the material as well as on the overlap between the optical and acoustic fields in the MWGs, $P_{\mathrm{IDT}}$ is the nominal radio-frequency $(\mathrm{RF})$ power applied to the interdigital transducer (IDT), and $\delta n_{\text {eff }}$ is the amplitude in the effective index modulation induced by the SAW. The latter is related to the effective index of the $j^{\text {th }} \mathrm{MWG}$ at a given time $(t)$ by [5]:

$$
n_{\text {eff }}(j)=n_{\text {eff }}^{0}+\kappa_{j}\left|\delta n_{\text {eff }}\right| \cos \left(\omega_{S A W} t\right)
$$

for $j=1,2$, where $n_{\text {eff }}^{0}$ is the unperturbed effective refractive index of the fundamental mode propagating in the MWGs, $\omega_{S A W}=2 \pi / T_{S A W}$ is the SAW angular frequency, with $\mathrm{T}_{\mathrm{SAW}}$ the SAW beating period, and $-1 \leq \kappa_{j} \leq 1$ are weight factors that account for the amplitude and the phase of modulation in the $j^{\text {th }}$ MWG by the travelling SAW generated by the IDT. In this case, two WGs are modulated with opposite phase and therefore, $\left(\kappa_{1}, \kappa_{2}\right)=(\mp 1, \pm 1)[5]$.

\section{$\underline{\text { Design and simulations }}$}

The devices were optimized to operate at $900 \mathrm{~nm}$. The width and length of the splitter and combiner MMI couplers are $13.9 \mu \mathrm{m}$ and $400.5 \mu \mathrm{m}$, respectively. The length of the center-fed splitter was calculated in order to only excite the even symmetric modes [6]. This results in more compact devices. The access WGs of both the splitter and combiner MMI couplers are tapered to avoid back-reflections into previous guiding sections of the device. The optimized tapered WGs are $\approx 230 \mu \mathrm{m}$-long, with $2.0 \mu \mathrm{m}$-width sections connecting the single-mode WGs to the MMI couplers. The IC of the device is also tapered, with a $4 \mu \mathrm{m}$-wide initial section, and a length of $\approx 300 \mu \mathrm{m}$. The response of the device was simulated using a finite difference beam propagation (BPM) method. Assuming either the transverse electric (TE) or the transverse magnetic (TM) polarizations at the IC, we tracked the propagation of the optical field throughout the device. Figure 2 shows the results for different times and TE polarization, taking $\delta n_{\text {eff }}=0.0019 . \mathrm{t}=0$ [Figure 2(a)] was chosen to coincide with an instant in which the device operates as in the absence of SAWs, with all the optical power delivered to the central WG $\left(\mathrm{OC}_{2}\right)$. For $\mathrm{t}=280 \mathrm{~ns}$ [Figure 2(b)], the optical power is evenly distributed within the OCs. Finally, when $\mathrm{t}=480 \mathrm{~ns}=$ $\mathrm{T}_{\mathrm{SAW}} / 4$ [Figure 2(c)], the light is distributed between the side WGs $\left(\mathrm{OCs}_{1,3}\right)$. 


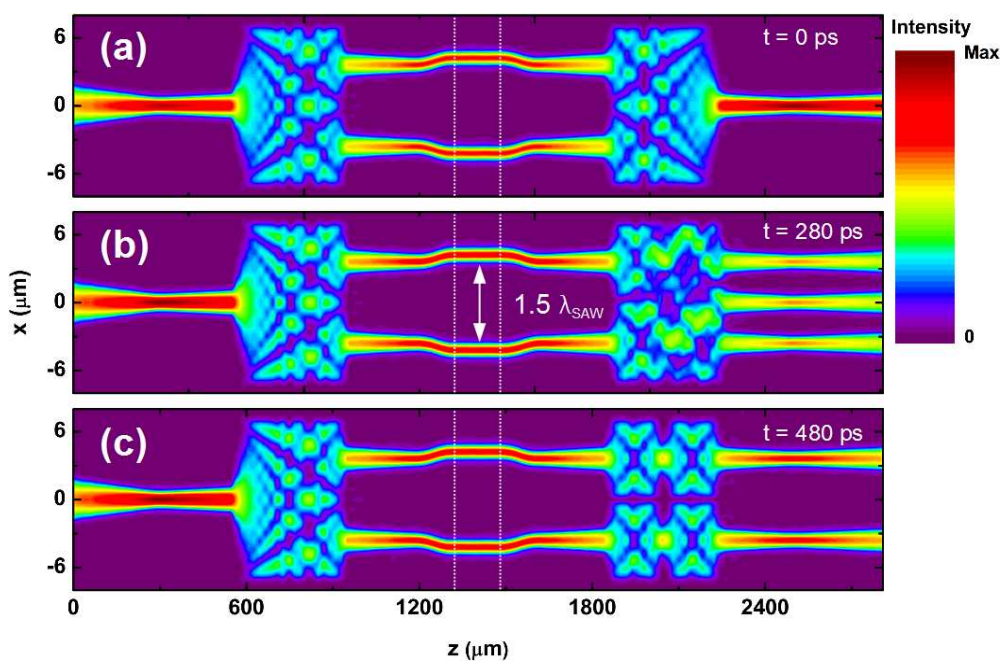

Figure 2. Color-scale maps of the optical intensity as the light propagates through the device for different times assuming $\delta n_{\text {eff }}=0.0019$ and TE polarization, according to BPM simulations. The area between the vertical dotted lines corresponds to the active region modulated by the SAW beam. The WGs in the modulated region are separated by $1.5 \lambda_{\mathrm{SAW}}$.

\section{DISCUSSION}

The modulator was fabricated in two steps using contact optical lithography on a sample grown by molecular beam epitaxy on a (001) GaAs wafer. The sample consists of a 300-nmthick GaAs film forming the guiding layer, deposited on a 1500 -nm-thick $\mathrm{Al}_{0.2} \mathrm{Ga}_{0.8} \mathrm{As}$ buffer layer. First, Ti/Al/Ti IDT in a split finger configuration for efficient SAW generation was fabricated using a lift-off process. The IDT was designed for an operation wavelength of $\lambda_{S A W}=5.6 \mu \mathrm{m}[2,4,5]$ (corresponding to a resonance frequency of approximately $520 \mathrm{MHz}$ ), with finger width and spacing of $700 \mathrm{~nm}$. A second step of plasma etching was employed to create the 137-nm-deep grooves delimiting the rib WGs of the device $[2,4,5]$. Top view micrographs of the fabricated devices can be seen in Figure 3(a).

The samples were optically characterized by coupling light into the input WG using a tapered optical fiber probe with a lensed tip. As light source, we used a superluminescent diode with peak emission centered at $920 \mathrm{~nm}$, with a full width at half maximum of approximately 40 $\mathrm{nm}$. The transmitted light was detected using a Si avalanche photodiode with a time resolution of 500 ps, synchronized with the RF signal driving the IDTs. Time-resolved transmission traces were recorded for different RF powers $\left(P_{I D T}\right)$ applied to the IDT, and light with TE polarization (similar behavior was observed for light with TM polarization). The total transmission is normalized to 1. At small $P_{I D T}$ [Figure 3(b), upper panel (i)], the light is modulated at the SAW fundamental frequency $\left(f_{S A W}\right)$, with the central WG contributing to nearly $80 \%$ of the total transmission. When $P_{I D T}$ increases, the presence of higher harmonics becomes evident. At $P_{I D T}=$ $68.3 \mathrm{~mW}$ [Figure 1(b), central panel (ii)], the modulation at the first harmonic is less intense with 


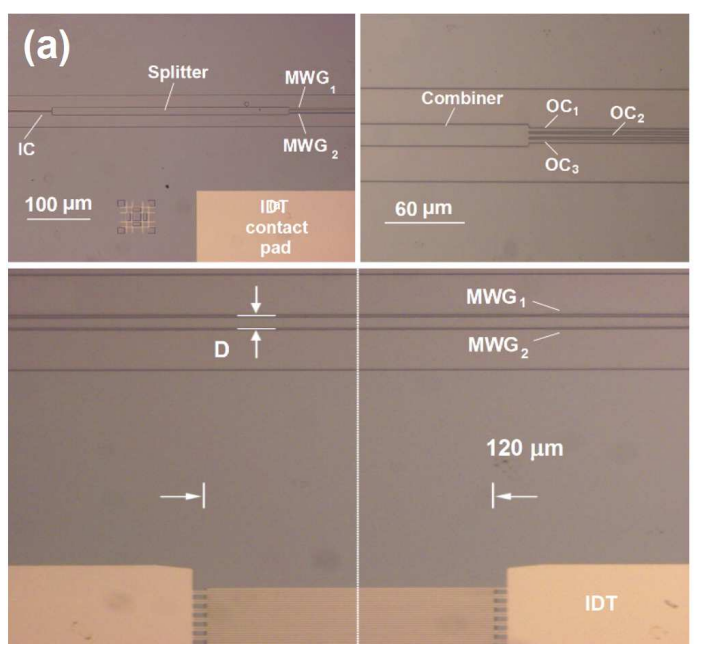

(b)

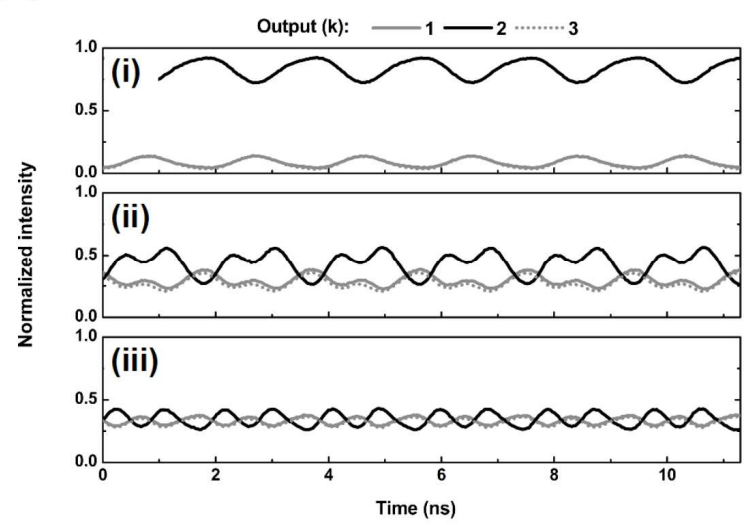

Figure 3. (a) Top view micrographs of splitter MMI coupler, the combiner MMI coupler and the OWGs, and the active region of the fabricated device. The separation between the WGs (D) was set to $1.5 \lambda_{S A W}$. (b) Time-resolved traces recorded for the light leaving the $\mathrm{OC}_{1}$ (dotted grey line), $\mathrm{OC}_{2}$ (solid black line) and $\mathrm{OC}_{3}$ (solid grey line), measured for the TE polarization and $\mathrm{RF}$ powers of (i) $P_{I D T}=6.8 \mathrm{~mW}$, (ii) $P_{I D T}=68.3 \mathrm{~mW}$ and (iii) $P_{I D T}=108.3 \mathrm{~mW}$.
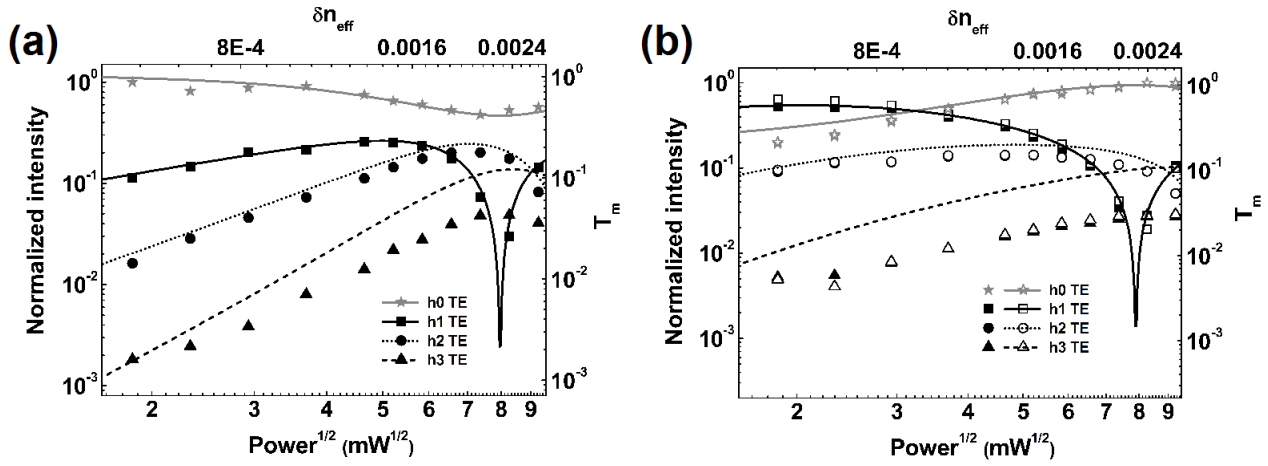

Figure 4. Experimental results (symbols) for the fast Fourier transform coefficients of the timeresolved traces for the mean value $(h 0)$, first, second and third harmonics $(h 1=519 \mathrm{MHz}, h 2=$ $1.03 \mathrm{GHz}$ and $h 3=1.55 \mathrm{GHz}$, respectively) as a function of $P_{I D T}$ (lower scale) for light with TE polarization. The solid lines correspond to the TE simulated results as a function of $\delta n_{\text {eff }}$ (upper scale), taking $\delta \varphi_{\mathrm{s}}=0.70 \mathrm{rad}$. (a) corresponds to results for $\mathrm{OC}_{2}$ and (b) to results for $\mathrm{OC}_{1}$ (full symbols) and $\mathrm{OC}_{3}$ (open symbols).

a simultaneous increase in the modulation at the second harmonic. At $P_{I D T}=108.3 \mathrm{~mW}$ [Figure 1(b), bottom panel (iii)], the light modulation is entirely dominated by the second harmonic. The harmonic content of the measured signals can be better appreciated by performing a Fast Fourier Transform (FFT) analysis of the experimental traces. Figure 4 displays the FFT coefficients for the mean value $(h 0)$ and the first three harmonic components ( $h 1, h 2$ and $h 3$, respectively) of the time-resolved experimental traces for $\mathrm{OC}_{2}$ [Figure 4(a)], and for $\mathrm{OCs}_{1,3}$ [Figure 4(b)], as a function of $P_{I D T}$ (lower scale) for light with TE polarization (very similar results were obtained 
for the TM polarization). The solid lines correspond to the calculated FFT coefficients as a function of $\delta n_{\text {eff }}$ (upper scale). A static phase shift $\delta \varphi_{\mathrm{s}}$ associated with asymmetries between the arms due to the fabrication process was used as a fit parameter in Figure 4, and determined to be $\delta \varphi_{\mathrm{s}}=0.70 \mathrm{rad}$. This static phase shift is close to $\pi / 4$, and is responsible for the light modulated at the first harmonic for the lowest $P_{I D T}$ in the time-resolved measured traces. By comparing the measured data with the simulated results, we determined $a_{p} \approx 17 \times 10^{-2} \mathrm{rad} / \mathrm{m} W^{1 / 2}$, which is close to previously obtained results $[2,4]$. There is an excellent agreement between the measurements on the fabricated device and the simulations as shown in Figure 4, where the mean value $h 0, h 1, h 2$, and $h 3$ intensities follow almost perfectly the calculated trend. Furthermore, the simulated results reproduce the vanishing of the first harmonic for high $P_{I D T}$ in the three OCs. The values obtained in the measurements of $h 2$ and $h 3$ intensities (which have an oscillation period of approximately $950 \mathrm{ps,} \mathrm{and} 600 \mathrm{ps}$, respectively) are considerably restricted by the time resolution of $500 \mathrm{ps}$, which limits the detection of higher harmonic components.

\section{CONCLUSIONS}

In conclusion, we have developed an acoustically driven photonic modulator based on a Mach-Zehnder interferometer that operates in the low GHz range. The lateral channels are $180^{\circ}-$ dephased with respect to the central one, whose phase and amplitude response can be changed by varying the applied acoustic power. The device was monolithically fabricated on (Al, Ga)As, although it can be easily implemented in other material platforms (InP, LiNbO3), or even in nonpiezoelectric materials, such as Si. In this case, SAWs can be generated by IDTs placed on a piezoelectric overlayer. Modulation at the second harmonic of the SAW driving frequency (1.03 $\mathrm{GHz}$ ) is accomplished. The large phase coherence of the SAWs allows for the modulation of several ridge or slot WGs using the same acoustic beam. The modulator can be used as a building block for more complex photonic functionalities.

\section{REFERENCES}

1. Jie Sun, E. Timurdogan, A. Yaacobi, E. Shah Hosseini and M. R. Watts, "Large-scale nanophotonic phased array," Nature, 493, 195-199 (2013).

2. M. M. de Lima Jr., M. Beck, R. Hey, and P. V. Santos, "Compact Mach- Zehnder acoustooptic modulator," Appl. Phys. Lett. 89, 121104 (2006).

3. M. Beck, M. M. de Lima, Jr., E. Wiebicke, W. Seidel, R. Hey, and P. V. Santos, “Acoustooptical multiple interference switches," Appl. Phys. Lett. 91, 061118 (2007).

4. A. Crespo-Poveda, R. Hey, K. Biermann, A. Tahraoui, P. V. Santos, B. Gargallo, P. Muñoz, A. Cantarero, and M. M. de Lima, Jr., "Synchronized photonic modulators driven by surface acoustic waves," Opt. Express. 21, 21669-21676 (2013).

5. A. Crespo-Poveda, A. Hernández-Mínguez, B. Gargallo, K. Biermann, A. Tahraoui, P. V. Santos, P. Muñoz, A. Cantarero, and M. M. de Lima, Jr., "Acoustically driven arrayed waveguide grating," Opt. Express. 23, 21213-21231 (2015).

6. L. B. Soldano and E. C. M. Pennings, "Optical multi-mode interference devices based on selfimaging: principles and applications,” J. Lightw. Technol. 13, 615-627 (1995). 